\title{
Effect of Carbon and Binder on High Sulfur Loading Electrode for Li-S Battery Technology
}

\author{
Ke Sun, ${ }^{\text {a }}$ Christina A. Cama, Jian Huang, ${ }^{\mathrm{c}}$ Qing Zhang, ${ }^{\mathrm{c}}$ Sooyeon Hwang, ${ }^{\mathrm{d}}$ Dong Su, ${ }^{\mathrm{d}}$ Amy \\ C. Marschilok, ${ }^{\text {b,c }}$ Kenneth J. Takeuchi, ${ }^{\text {b,c }}$ Esther S. Takeuchi ${ }^{\text {b,c, e }}$ and Hong Gan ${ }^{\text {a,z }}$ \\ ${ }^{a}$ Sustainable energy Technologies Department, Brookhaven National Laboratory, Upton, NY 11973 \\ ${ }^{b}$ Department of Chemistry, Stony Brook University, Stony Brook, NY 11794 \\ ${ }^{c}$ Department of Materials Science and Engineering, Stony Brook University, Stony Brook, NY 11794 \\ ${ }^{d}$ Center for Functional Nanomaterials, Brookhaven National Laboratory, Upton, NY 11973 \\ ${ }^{e}$ Energy Sciences Directorate, Brookhaven National Laboratory, Upton, NY 11973
}

\begin{abstract}
For the Lithium-Sulfur (Li-S) battery to be competitive in commercialization, it is requested that the sulfur electrode must have deliverable areal capacity $>8 \mathrm{mAh} \mathrm{cm}^{-2}$, which corresponds to a sulfur loading $>6 \mathrm{mg} \mathrm{cm}^{-2}$. At this relatively high sulfur loading, we evaluated the impact of binder and carbon type on the mechanical integrity and the electrochemical properties of sulfur electrodes. We identified hydroxypropyl cellulose (HPC) as a new binder for the sulfur electrode because it offers better adhesion between the electrode and the aluminum current collector than the commonly used polyvinylidene fluoride (PVDF) binder. In combination with the binder study, multiple types of carbon with high specific surface area were evaluated as sulfur hosts for high loading sulfur electrodes. A commercial microporous carbon derived from wood with high pore volume showed the best performance. An electrode with sulfur loading up to $10 \mathrm{mg} \mathrm{cm}^{-2}$ was achieved with the optimized recipe. Based on systematic electrochemical studies, the soluble polysulfide to insoluble $\mathrm{Li}_{2} \mathrm{~S}_{2} / \mathrm{Li}_{2} \mathrm{~S}$ conversion was identified to be the major barrier for high loading sulfur electrodes to achieve high sulfur utilization.
\end{abstract}

Key Words: Li-S battery, binder, carbon types, particle size, pore volume, electrode loading.

${ }^{2} E-m a i l:$ hgan@bnl.gov

(C) 2017. This manuscript version is made available under the Elsevier user license

http://www.elsevier.com/open-access/userlicense/1.0/ 


\section{Introduction}

The lithium-sulfur battery (Li-S) has been one promising alternative electrochemical energy storage system with high energy density [1,2], compared with the current market-dominating Li-ion batteries based on intercalation chemistries. It has a theoretical energy density of $2600 \mathrm{Wh} \mathrm{kg}^{-1}$, about 6 times that of the Li-ion battery with $\mathrm{LiCoO}_{2}$ and graphite electrodes. Additionally, sulfur's abundance on earth enables the Li-S battery to be a low-cost system.

In spite of these advantages, the Li-S battery system under development still suffers from low energy utilization and efficiency due to fundamental material properties such as low electronic conductivity of both sulfur and lithium sulfide $\left(\mathrm{Li}_{2} \mathrm{~S}\right)$, active material dissolution, and the well-known shuttling effect [13]. The major contributor to the latter two issues has been identified as highly soluble polysulfides $\left(\mathrm{S}_{\mathrm{n}}{ }^{2-}\right.$ $2<\mathrm{n}<8)$ generated during the initial lithiation process of sulfur. These soluble species diffuse out of the cathode network and become inaccessible to the cathode, thereby, inducing irreversible capacity loss. Additionally, the polysulfides consume extra energy during recharge as they form a secondary redox cycle between the anode and cathode resulting in a high degree of self-discharge. With the current electrolyte system, it is difficult to stop the sulfur dissolution and shutting process [4]. One possible strategy to solve these issues is to confine polysulfides in the cathode and slow down their outward propagation. Porous carbon architectures possessing enormous surface area offer an abundance of sites for sulfur adsorption. There have been multiple instances in the literature that have demonstrated the ability of carbon to accommodate large amounts of sulfur and effectively immobilize the soluble species during operation of the cathode [5-10]. In addition, carbon's decent electronic conductivity also make it an ideal choice amongst the different porous materials [11-13].

Despite these encouraging improvements on material properties, the commercialization of the Li-S battery is still hindered by some important practical issues, such as final pack-level energy density, scaleup difficulty, high material and processing cost, and competitiveness with current technology. Recently, it has been predicted in a numerical cost analysis that capacity loading of sulfur electrode has to be higher than $8 \mathrm{mAh} \mathrm{cm}^{-2}$ in order to be competitive for the market [14]. Unfortunately, except for a few cases that applied very sophisticated methods [15-20], reports on sulfur electrodes with high capacity loading are rare. One of the main reasons for the scarcity of high areal capacity sulfur electrodes is the difficulty in obtaining thick, mechanically robust sulfur electrodes. As mentioned above, confining polysulfides to achieve stable cycling capability demands the aid of a porous carbon host. To make this route effective, a low S/C ratio has to be applied and the final content of sulfur in the electrode is only about $60 \%$. In this case, the final electrode thickness should be at least 150 microns (sulfur loading of $8 \mathrm{mg} \mathrm{cm}^{-2}$ ) to achieve $8 \mathrm{mAh} \mathrm{cm}^{-2}$ [21]. Compared with the Li-ion battery, whose electrodes are generally 100 microns or below in thickness [22, 23], Li-S battery experiences more difficulty in electrode processing. This is further exacerbated by the fact that most of the effective carbon hosts are in the form of nano-structured carbon such as carbon nanotube, graphene oxide and porous carbon nanoparticles. These small particles are detrimental to the final density and mechanical stability of the electrode. Correspondingly, it has recently been shown that gluing small microporous carbon nano-particles into secondary agglomerations of $\sim 5-10$ microns size can help to improve the mechanical property of the electrode dramatically and enable the preparation of much thicker electrodes [24, 25]. This also suggests that the carbon hosts with bigger primary particle sizes may help to reduce the difficulty with the manufacturing of thick sulfur electrodes.

ZE-mail: hgan@bnl.gov 
Besides the particle size of carbon hosts, binder is particularly important to achieve high loading electrode because it is controlling both adhesion at the interface between the current collector and the electrode body, and cohesion among the electrode components. PVDF is still the most common binder used for sulfur electrodes; however, it does not seem to provide sufficient adhesion to the aluminum current collector. Aqueous based binders such as carboxymethyl cellulose (CMC) is reported to enhance adhesion due to possible interactions between their hydroxyl groups and the native hydrophilic oxide surface layer on aluminum foil [24, 26]. However, it is difficult to remove moisture from sulfur electrodes with hydrophilic binders because of sulfur's volatile nature. The residual water content might be a threat to both polysulfide species in the electrolyte and lithium anode, both of which react irreversibly with $\mathrm{H}_{2} \mathrm{O}$. Similar to CMC, hydroxypropyl cellulose (HPC) is a member of the cellulose family and can be dissolved in non-aqueous solvents such as 1-methyl-N-pyrollidone to allow waterless processing. The use of HPC as binder in Li-S batteries has not been reported.

In this work, we investigate the impact of the nature of binder and carbon on the mechanical integrity and the electrochemical properties of sulfur electrodes in order to develop a high sulfur loading Li-S battery. We systemically studied different types of carbon with various particle sizes, pore volumes and specific surface areas, to achieve the sulfur electrode loading up to $12 \mathrm{mAh} \mathrm{cm}^{-2}$. We found that microporous carbon derived from natural sources with the primary particle size above 10 microns indeed enables the facile processing of thick sulfur electrodes stable enough for cell processing and assembly, which is superior to nanosized carbon particles such as Super C65 and Ketjenblack. Among the different physical properties, large pore volume is also identified as the key parameter in the selection of the ideal porous carbon matrix for sulfur electrodes with superior electrochemical performance. On the other hand, a new HPC binder has also been identified to improve sulfur electrode-current collector adhesion.

\section{Experimental}

\subsection{Material and Material Characterization}

Three types of microporous carbon including A5562, A5583 and A5597 were provided by Asbury Carbons Inc. They are documented to be derived from coconut shell (A5562), coal (A5583) and wood (A5597), respectively. They should be manufactured by a high temperature pyrolysis of their respective natural precursors under inert atmosphere, but the exact synthesis condition is not available. Ketjenblack (EC-330JMA) was obtained from AkzoNobel and Super C65 was provided by Imerys Graphite \& Carbon Inc. All of them were dried under vacuum at $150^{\circ} \mathrm{C}$ for 24 hours before use and stored in a dry room with due point below $-40^{\circ} \mathrm{C}$ to prevent rehydration. Scanning electron microscopy (JEOL 7600F) and transmission electron microscopy (JEOL 2100F) were applied to characterize the morphology of these 5 candidates. Their BET surface area, pore volume and pore size were measured with a Nova Quantachrome surface area and pore size analyzer using nitrogen gas sorption techniques. TGA/DSC measurements were done using a TA Instruments Q600 SDT simultaneous thermal analyzer. Samples were heated under nitrogen atmosphere to $580^{\circ} \mathrm{C}$ at a rate of $10^{\circ} \mathrm{C} / \mathrm{min}$. The water content was estimated from the thermal analysis using the mass loss exhibited between room temperature and $400^{\circ} \mathrm{C}$. Additional water composition analyses were done using a Mettler - Toledo Karl - Fischer (KF) Coulometer C20. For each Asbury brand carbon, $10 \mathrm{mg}$ were stirred in $5 \mathrm{~mL}$ of anhydrous Acetonitrile (Honeywell) for 15 minutes in a dry room with average due point of $-60^{\circ} \mathrm{C}$. The mixture was filtered to collect the solvent,

'E-mail: hgan@bnl.gov 
then, aliquots of the acetonitrile were used for KF analyses. The KF titrations were done with Hydranal Coulomat AG (Sigma Aldirch) solution as the anolyte. The two binders polyvinylidene fluoride (PVDF, $\mathrm{MW}=600,000)$ and hydroxypropyl cellulose (HPC, MW=1,000,000) were both obtained from Alfa Aesar. They were dried under vacuum at $80^{\circ} \mathrm{C}$ for 24 hours before use.

\section{2. $\quad$ Binder and Electrode Adhesion Study}

Both HPC and PVDF were used as binder in electrode slurry with 50wt.\% sulfur (Alfa Aesar), 40wt.\% Super C65 and 10wt.\% binder (compositions 1 and 2, Table 1). Different components were mixed in NMethyl-2-pyrrolidone (NMP, Sigma Aldrich) and casted onto an aluminum foil as current collector with a doctor blade. After drying of the electrodes first at room temperature for 24 hours and another 24 hours at $50^{\circ} \mathrm{C}$ under dry air flow (dew point $<-40^{\circ} \mathrm{C}$ ), a tape test was performed on them. A piece of scotch type tape was applied onto each electrode with an area of $1.27 \mathrm{~cm}^{2}$ defined by a circular mask and a load of $1.2 \mathrm{~kg}$ was applied on this area for $30 \mathrm{~s}$. The initial average loading and the amount of material loss were both measured by a digital balance. Charge-Discharge measurements of the two electrodes were done in 2032 coin cells using the same method mentioned in the following text at a current density equivalent to 0.2C. After 100 cycles, the cells were open and the cycled electrodes were both soaked in 1, 2-Dioxlane (Sigma Aldrich) to check the electrode adhesion again with different binders.

\subsection{Carbon Candidates Evaluation}

A sulfur-carbon composite of each carbon was prepared by heating a sulfur-carbon mixture with a 3:1 weight ratio at $155^{\circ} \mathrm{C}$ for 24 hours in a sealed Teflon container. Each obtained sulfur-carbon mixture was crushed and milled in a mortar to reduce the size of the agglomerates to enable homogeneousness in the final electrode slurry. Electrodes for each carbon were prepared by mixing different components in NMP, and casting onto aluminum foil. The electrode composition for the initial carbon selection was sulfur:carbon:Super C65:HPC = 62:21:9:8 by weight (composition 3, Table 1). Additional Super C65 is needed to provide a better electron percolation network.

2032 coin cells were assembled by using electrodes prepared above as the working electrode and a lithium disk as both counter and reference electrodes, with 2 layers of Celgard (2325) separator in between to prevent shorting. The electrolyte used for testing was LiTFSI $(1.0 \mathrm{M})$ dissolved in 1,2dimethoxyethane (DME) and 1,3-dioxolane (DOL) (1:1 ratio, by volume) obtained from BASF. 1wt.\% of $\mathrm{LiNO}_{3}$ was added to enhance Coulombic efficiency. For coin cells used in this study, the amount of electrolyte was kept at $80 \mu \mathrm{L}$ for all cells. We found that lower electrolyte filling volume introduces severe cell-to-cell performance variation. On the other hand, when higher electrolyte volume is used, part of the electrolyte can be squeezed out of the cell during the cell-crimping step in coin cell preparation, which makes the quantitative amount of control of the electrolyte difficult. Electrochemical measurements were conducted at room temperature using a Bio-logic (VMP3) battery cycler. Cells were cycled galvanostatically at $0.2 \mathrm{C}$ between $1.8 \mathrm{~V}$ and $2.6 \mathrm{~V}$ versus lithium. Each charge also included a constant voltage step until the current fell below $0.05 \mathrm{C}$.

\subsection{Sulfur Loading Effect Study}

The electrode composition for the sulfur loading effect study was sulfur:A5597:Super C65:HPC:PVDF=62:27:3:4:4 by weight (composition 4, Table1). Such sulfur electrodes with different

ZE-mail: hgan@bnl.gov 
loading levels were discharged at $0.08 \mathrm{~mA} \mathrm{~cm}, 0.08 \mathrm{~mA} \mathrm{~cm}{ }^{-2}, 0.16 \mathrm{~mA} \mathrm{~cm}{ }^{-2}, 0.31 \mathrm{~mA} \mathrm{~cm}$, and 0.62 $\mathrm{mA} \mathrm{cm}{ }^{-2}$ respectively for the first 5 cycles, and the charge current correspondingly was $0.08 \mathrm{~mA} \mathrm{~cm}$, $0.08 \mathrm{~mA} \mathrm{~cm}{ }^{-2}, 0.16 \mathrm{~mA} \mathrm{~cm}{ }^{-2}, 0.31 \mathrm{~mA} \mathrm{~cm}^{-2}$, and $0.62 \mathrm{~mA} \mathrm{~cm}$, each with a constant voltage charge process until the current dropped below $0.08 \mathrm{~mA} \mathrm{~cm}^{-2}$. The corresponding C-rate levels are shown in Table 2 based on sulfur's theoretical capacity (1674 mAh/g sulfur).

During pulse testing, cells of 4 different loading levels were discharged intermittently to various depths of discharge at a lower current density $\left(0.08 \mathrm{~mA} \mathrm{~cm}^{-2}\right)$ and a high current density $\left(0.62 \mathrm{~mA} \mathrm{~cm}{ }^{-2}\right)$ pulse was applied to them following each of the sequences in order to measure DC resistance of the cell $R_{d c}$ as a function of depth of discharge. Between each intermittent discharge and the ensuing pulse, each cell was also put into open circuit period for 15 minutes. Although 15 minutes is not enough time to achieve real equilibrium in this system because of the presence of polysulfides and the shuttling effect, it does provide a reasonable time window for the system to relax from the discharge voltage bias. The test was allowed to proceed until the voltage reached $1.6 \mathrm{~V}$ during the $0.08 \mathrm{~mA} \mathrm{~cm}^{-2}$ constant current discharge sequence. The DC resistance was calculated by using the voltage difference between the last point of the open circuit process of current sequence and the last point of $2.5 \mathrm{~s}$ pulse process of the next one, divided by the current $0.8 \mathrm{~mA}\left(0.62 \mathrm{~mA} \mathrm{~cm}^{-2}\right)$.

The composition of sulfur electrode with $\mathrm{TiS}_{2}$ additive was $\mathrm{S}: \mathrm{A} 5597:$ Super C65:TiS 2 :HPC:PVDF = 56:16:11:9:4:4 by weight (composition 5, Table 1). $\mathrm{TiS}_{2}$ was added during the slurry mixing process, which was after the thermal fusion step of sulfur and A5597. It was cycled with the same protocol used for the loading study aforementioned. The testing equipment and cell designs in these 3 experiments were the same as described in the carbon selection study.

\section{Results and Discussion}

\subsection{Binder Performance}

Two binders, PVDF and HPC, were tested for their adhesion to the aluminum foil current collector. Figure 1a contains two photos of local areas of electrodes prepared with either 10\% PVDF binder or $10 \%$ HPC binder before and after a tape test. It can be clearly seen that the electrode with the PVDF binder experienced significant damage but the one with HPC binder remained largely intact. In the comparison plot shown in Figure 1b, this difference is quantitatively expressed and it is evident that HPC binder provides much better adhesion between the aluminum current collector and the electrode.

Despite the poor adhesion exhibited by the PVDF electrodes, there is not much difference in cycling performance, as shown in Figure 1c. After 100 cycles between $1.8 \mathrm{~V}$ and $2.6 \mathrm{~V}$ at $0.2 \mathrm{C}$ rate, the cells were disassembled and the electrodes were extracted and soaked in 1,2-Dioxlane (Sigma Aldrich). As depicted in Figure 1d, the PVDF electrodes delaminate from the current collector easily while the HPC electrodes remain adhered to the aluminum foil current collector. This means strong adhesion of HPC binder is still preserved after cycling. Since the coin cells use a spring to maintain the cell stack pressure, the impact of the electrode to current collector adhesion on cell electrochemical performance may not be obvious. However, with pouch cells, there is no external force for the cells to maintain the cell stack pressure. The

E-mail: hgan@bnl.gov 
delamination of the electrode from the current collector (Figure 1d) could cause significant increase in the cell internal resistance, leading to degraded long-term cell cycling performance.

On the other hand, there is indeed some negative impact on the power capability of the sulfur electrode when PVDF is replaced with HPC, as it can be seen in Figure 1e. After cycling at a higher discharge rate of $1 \mathrm{C}$, the HPC electrode exhibited less capacity than the corresponding PVDF electrodes. This is probably due to HPC's stronger affinity for the surface of carbon fillers, and as a result HPC tends to coat and cap the conductive surface of carbon more efficiently than PVDF, which slightly undermines the conductive network. This is indirectly supported by a comparison of the stability of sulfur electrode slurries processed with these two binders. In Figure 1f, the photos of two different, thoroughly mixed, slurries after 2 hours of settling are shown. Most of the carbon particles sank to the bottom of the vial with PVDF and a transparent supernatant is left, while in the HPC sample the carbon is still well dispersed and the solution appears to be very dark and concentrated [27].

Considering these observations, it is suggested that HPC could be used as the sole binder for a low loading sulfur electrode or one of the dual binders for a heavily loaded electrode when the electronic conduction becomes highly important, as it is done in this study.

\subsection{Carbon Characterization}

SEM images of A5562, A5583, A5597, Ketjenblack and Super C65 are shown in Figure 2 a-e. From these images, it is evident that all of the different types of microporous carbon from Asbury have primary particle sizes above 10 microns, much bigger than the Ketjenblack and Super C65, both of which are on average only $50 \mathrm{~nm}$ in diameter, as shown in the TEM micrographs in Figure $2 \mathrm{f}-\mathrm{j}$. It is hopeful that these micron-sized microporous carbons can deliver better packing efficiency in the electrode processing, without sacrificing too much of their absorbing ability for sulfur.

It has been generally acknowledged that high surface area is the most important factor for considering any form of carbon as a component in a sulfur electrode matrix. The surface area should be sufficient in providing enough active surface sites to adsorb and immobilize polysulfides. In one study, microporous carbon was prepared from the pyrolysis of apricot shells. The resulting carbon exhibited high surface area of $2269 \mathrm{~m}^{2} \mathrm{~g}^{-1}$ and a pore volume of $1.05 \mathrm{~cm}^{3} \mathrm{~g}^{-1}$, as measured by BET nitrogen adsorption data. The high surface area and pore volume lead to the homogenous distribution of sulfur [28].

Although BET surface area is provided by each manufacturer, it is still measured here for each carbon using the same equipment and under the same conditions to offer a fair comparison. As shown in Figure 3a, all of the carbons possess very high specific surface area in the neighborhood of $1000 \mathrm{~m}^{2} \mathrm{~g}^{-1}$, except for Super C65 that shows a surface area less than $60 \mathrm{~m}^{2} \mathrm{~g}^{-1}$. Also shown in Figure 3a is the specific pore volume. Only Ketjenblack and A5597 showed values in the order of $1.0 \mathrm{~cm}^{3} \mathrm{~g}^{-1}$, while less than $0.1 \mathrm{~cm}^{3}$ $\mathrm{g}^{-1}$ was measured for A5562, A5583 and Super C65.

In addition to the surface area and pore volume analyses, both TGA/DSC and Karl-Fisher Titration methods were used to analyze the water content of the microporous carbons. Interestingly, both Ketjenblack and Super C65 did not exhibit any significant mass loss upon heating from room temperature to $580^{\circ} \mathrm{C}$ under nitrogen atmosphere. The three Asbury carbons, on the other hand, showed significant mass loss due to water evaporation; A5597 in particular, exhibited the largest mass loss, 21\% and A5562

E-mail: hgan@bnl.gov 
exhibited only $6.7 \%$ loss. The mass loss observed during heating under nitrogen was attributed to surface water. The water content was verified using the Karl-Fisher titration method, as described in Figure $3 b$. According to Figure 3b, the TGA/DSC and KF data agree well.

\subsection{Sulfur-Carbon Composite Study}

In order for each porous carbon to work efficiently as the sulfur host, a thermal sulfur impregnation has to be applied before the electrode slurry processing. During this process, the carbon and sulfur are heated in an oven at $155^{\circ} \mathrm{C}$ for 24 hours. The weight change of the mixture before and after thermal treatment indicates that no sulfur was lost in all cases and sulfur seems to be fully incorporated into the different carbon hosts. However, significant differences in morphology emerge in the different carbon-sulfur mixtures after the thermal process, as depicted in Figure 4a. In particular, two different behaviors can be observed. For A5562 and A5583, the thermal treatment leads to a single monolith, which is highly rigid and requires significant force to break apart in a mortar. Meanwhile, for Super C65, Ketjenblack and A5597, the sulfur-carbon mixture remains a fine powder after thermal treatment. The former behavior is interpreted as the inability of the porous carbon to fully accommodate sulfur into its interior space and the excess sulfur left binds the carbon particles together during cooling. Thus, the latter is inferred as having the better capability for sulfur absorption.

SEM/EDS mapping micrographs in Figure 4b-f provide some evidence to this explanation. There is a significant amount of inhomogeneity in the sulfur and carbon distributions, which can be clearly observed for the S-C composites A5562 and A5583. However, better overlap is seen in Ketjenblack and A5597, indicating there is stronger sulfur incorporation in these carbons than the former two.

Considering both the distribution of sulfur in the carbon and the measured physical properties of the carbons, a positive correlation seems to exist between the specific pore volume and the capability of sulfur absorption. The carbons with small pore volumes could not occupy much sulfur, but the carbons possessing larger pore volumes were capable of accommodating more sulfur. Super C65 is a special case here: it has the least pore volume but does not form large clumps as A5562 and A5583 do during sulfur impregnation. This might be related to the particle size effect. Super C65 has a small particle size ( $\sim 50$ $\mathrm{nm}$ ), thus, its packing efficiency decreases compared to the microporous carbon particles that are tens of microns in size. The poorly packed Super C65 ensemble contains a huge volume fraction of voids, which is enough to accommodate all of the sulfur; thereby, preventing the collapse of the mixture into a single hard piece as with A5562 and A5583. Despite the efficient accommodation of sulfur, inhomogeneous sulfur inclusions can still be distinguished as shown in Figure 4f, unlike the homogeneous sulfur adsorption shown in Figures $4 d$ and $4 \mathrm{e}$ for Ketjenblack and A5597. Thus, this study shows that both pore volume and particle size are necessary factors for considering a carbon additive for the sulfur electrode.

After the different Sulfur-Carbon mixtures were obtained, the electrodes were processed with additional Super C65 carbon and HPC binder with varying thicknesses. The photos of these electrodes with different carbons, but similar sulfur loadings of $1.25 \mathrm{mg} \mathrm{cm}^{-2}$ and $4.0 \mathrm{mg} \mathrm{cm}^{-2}$ are shown in Figure 5a-e. It is evident that at higher loading, electrodes with Super C65 and Ketjenblack already exhibit significant delamination and cracking; on the other hand, the 3 Asbury microporous carbons form more robust electrodes at this loading level. Similar behavior has been observed in References [24] and [25], and this again exemplifies the advantage of carbon with bigger primary particle sizes in high loading electrode processing.

E-mail: hgan@bnl.gov 
In order to compare the effectiveness of the different carbons as sulfur hosts, cycling tests were performed on electrodes with lower loading of $1.25 \mathrm{mg} \mathrm{cm}^{-2}$, at which electrodes of all carbon still have good mechanical integrity. The first discharge voltage profiles for all 5 electrodes were plotted in Figure $5 f$. The Ketjenblack electrode has the highest capacity of $832 \mathrm{mAh} \mathrm{g}^{-1}$; Super C65 and A5597 have similar capacities close to $750 \mathrm{mAh} \mathrm{g}^{-1}$; the voltage profiles of these 3 carbons all exhibit a sloped region between $2.35 \mathrm{~V}$ and $2.05 \mathrm{~V}$ and a plateau at $2.05 \mathrm{~V}$, which is commonly observed for the Li-S cell at discharge. A5562 and A5583 electrodes both deliver much less capacity $\left(\sim 400 \mathrm{mAh} \mathrm{g}^{-1}\right)$ compared to Ketjenblack, Super C65 and A5597 carbons. The most notable difference can be seen in the length of the discharge plateau at $2.05 \mathrm{~V}$, which is significantly shorter for the cells prepared with A5562 and A5583 electrodes. The cycling performance of 5 electrodes over 100 cycles is also shown in Figure $5 \mathrm{~g}$. The Ketjenback electrode shows the best performance in terms of both capacity retention and sulfur utilization; A5597 delivered a little less capacity than Ketjenblack, but its cycling is also quite stable such that after 100 cycles there is not much fading. The Super C65 electrode started with almost the same capacity as A5597, but experienced a steady fade towards $384 \mathrm{mAh} \mathrm{g}^{-1}$ in 100 cycles. Both A5562 and A5583 showed capacities lower than $320 \mathrm{mAh} \mathrm{g}^{-1}$ consistently throughout the test.

Based on the above comparison, A5597 is the only candidate among the 3 microporous carbons to achieve acceptable electrochemical performance when serving as a sulfur host. The main reason that A5562 and A5583 do not work well as a carbon matrix for the sulfur electrode is due to their failure to absorb enough sulfur in the thermal fusion process, which has been ascribed to their low specific pore volume. The reason why A5597 possesses a higher pore volume after pyrolysis than the other two candidates is probably the special microstructure of wood.

The sulfur electrodes with Ketjen Black and Super C65 provide good electrochemical cell performance in terms of sulfur utilization and cycle life (Figure 5g). However, with these carbon materials, a high sulfurloading electrode cannot be achieved under our electrode slurry casting conditions due to their small particle size. Others have also reported the same observation for Ketjen Black [24, 25]. The carbon materials will not be practical or useful for Li-S battery technology commercialization without being able to achieve the high sulfur loading [14]. The coin cells with A5597 show better electrochemical cycling performance than cells with Super C65 and very close to that of cells with Ketjen Black after 100 cycles (Figure 5g). Therefore, the A5597 carbon clearly out-performed Ketjen Black and Super C65 on its overall performances by considering both mechanical and electrochemical properties.

Additional experiments were done to optimize the electrode processing of electrodes with A5597 as the major carbon component within the sulfur electrode. Because of the complex interactions between different components in the electrode slurry and the lack of theoretical guidance, the process is completely empirical. In this study, the final recipe achieved is $62 \mathrm{wt} . \%$ sulfur, $27 \mathrm{wt} . \%$ A5597, 3wt.\% Super C65, 4wt.\% PVDF and 4wt.\% HPC in the electrode slurry. This recipe is arrived through consideration of all aspects including energy density, electronic percolation, surface smoothness and mechanical robustness and flexibility. Ideally, it will be desirable to maximize sulfur content to increase energy density, use more A5597 than Super C as carbon filler to allow the coating of a thick electrode, and use HPC as sole binder to improve mechanical stability. However, all of these measures will increase the electronic resistance of the electrode, and completely eliminating Super $\mathrm{C}$ will also lead to the electrodes' surface roughness that might penetrate the porous separator. The above formulation is a

E-mail: hgan@bnl.gov 
preliminary compromise achieved between the energy density and power capability, in addition to mechanical considerations.

\subsection{Sulfur Loading and Electrochemical Performance}

Sulfur electrodes of various sulfur loadings using the above-mentioned final recipe were tested in 2032 coin cells to evaluate the sulfur loading effect on the electrochemical performance at multiple discharge current densities. The areal capacity and sulfur based specific capacity are separately plotted as a function of both current density and sulfur loading as depicted in Figures $6 \mathrm{a}$ and $6 \mathrm{~b}$. The linear relationship is preserved at a low current density of $0.08 \mathrm{~mA} \mathrm{~cm}{ }^{-2}$ for areal capacity delivered and areal sulfur loading, and very high areal capacity up to $12 \mathrm{mAh} \mathrm{cm}^{-2}$ was observed. This was also maintained during the second cycle with the same current density, with about $15 \%$ capacity loss. Increasing the current density lead to less efficient capacity delivered. Interestingly, it is the higher loading electrodes that start to experience this power limitation first. Notably, at the same current density $0.16 \mathrm{~mA} \mathrm{~cm}{ }^{-2}$, the high loading electrodes are discharged at a much lower C-rate than low loading ones, while the proportion of theoretical capacity delivered is the lowest for the highest loaded electrode $10.32 \mathrm{mg} \mathrm{cm}^{-2}$, as described in Table 2 and Figure 6b. This naturally suggests that Li-ion or electron transport becomes the rate limiting step as the thickness of the electrode is increased, which renders the additional sulfur content and associated thickness a burden for transport instead of a capacity source for the electrode. The dependence of sulfur utilization on loading follows the same trend as the current density is further increased, with the onset of inefficient capacity delivery shifts toward lower loading in Figures $6 \mathrm{a}$ and $6 \mathrm{~b}$.

The observation above indicates that the transport processes potentially become the rate-limiting factor as sulfur loading increases. Figures $6 \mathrm{c}$ and $6 \mathrm{~d}$ display the voltage profiles for sulfur electrodes of different loading levels at the lowest current density $0.08 \mathrm{~mA} \mathrm{~cm}^{-2}$ and the highest current density $0.63 \mathrm{~mA} \mathrm{~cm}^{-2}$. At the lowest current density, all loading levels deliver over $50 \%$ of the theoretical capacity; additionally, all of the voltage profiles clearly depict both the sloped discharge region between $2.3 \mathrm{~V}-2.1 \mathrm{~V}$ and the plateau discharge region at $\sim 2.1 \mathrm{~V}$, which assumes over $70 \%$ of the total capacity delivered, typical of Li-S cell. At the highest current density $0.63 \mathrm{~mA} \mathrm{~cm}$, only the lowest loading electrodes deliver acceptable capacity, while all other electrodes still seem to deliver similar capacities to the lowest loading ones in the sloped region (with $1.9 \mathrm{~V}$ as cut-off voltage). However, the plateau region fails to appear in these cases, which accounts for the majority of the capacity deficit. The failure of higher loading sulfur electrodes to deliver the plateau region suggests that there is a major energy barrier to be overcome before the plateau discharge can be initiated. Sometimes this barrier can be overcome with an activation process, in the form of a initial voltage dip followed by a slow voltage recovery process to reach a plateau discharge region, as it is shown in Figure 6e for $8.90 \mathrm{mg} \mathrm{cm}^{-2}$ electrode at $0.16 \mathrm{~mA} \mathrm{~cm}^{-2}$. However, at higher current densities the activation process could not be done without going to voltage cut offs lower than $1.7 \mathrm{~V}$.

\subsection{Mechanism Investigation}

To obtain a better understanding of above phenomenon, a DC pulse current test was applied to sulfur electrodes with different loadings at different depths of discharge (DOD) to measure the DC resistance evolution. The DOD is defined with reference to the accumulative capacity delivered at the lower current discharge cut-off voltage 1.6V. As it is shown in Figure 6f, all cells, except the cell with the highest sulfur

loading $11.00 \mathrm{mg} \mathrm{cm}^{-2}$, experience a gradual increase of DC resistance as the cell is discharged until a local maximum is reached at $20-25 \%$ DOD. The DC resistance then starts to decrease after the local

E-mail: hgan@bnl.gov 
maximum and reach a minimum at 50\% DOD, then gradually increases until discharge is completed at $1.6 \mathrm{~V}$. It needs to be emphasized that these local maximum points near $20 \%-25 \%$ of discharge all approximately correspond to the transition points from sloped discharge region to plateau discharge region for these sulfur electrodes. Another observation to be noted is that the higher the loading, the larger the difference between the local maximum around 20-25\% discharge and the initial DC resistance at 30 $\mathrm{mAh} \mathrm{g}^{-1}$. The overlap of the local DC resistance maximum and the transition point from sloped to plateau discharge supports the existence of an energy barrier at this point, and the higher resistance maximum values for higher loading sulfur electrodes can qualitatively demonstrate the higher energy barrier experienced by the thicker electrode. Since the transition point is generally correlated with the nucleation process of solid $\mathrm{Li}_{2} \mathrm{~S}_{2} / \mathrm{Li}_{2} \mathrm{~S}$, it is natural to attribute the energy barrier to the $\mathrm{Li}_{2} \mathrm{~S}_{2} / \mathrm{Li}_{2} \mathrm{~S}$ nucleation energy barrier. However, it is difficult to exclude the increase in electrolyte viscosity in cells with different levels of sulfur loading, which can cause the resistance to increase [21]. In this work, all cells were filled with the same amount of electrolyte $\sim 80 \mu \mathrm{L}$, and it is expected that any higher sulfur loading electrode will also generate higher concentrations of polysulfides dissolved in the electrolyte at the same depth of discharge of sulfur. Higher concentrations of polysulfides will lead to higher electrolyte viscosities and higher resistances, which is also consistent with the results of Figure 6f. Additional studies are required to distinguish the two possible mechanisms for this high-energy barrier at the transition point.

If $\mathrm{Li}_{2} \mathrm{~S}_{2} / \mathrm{Li}_{2} \mathrm{~S}$ nucleation is actually the obstacle for the functioning of high loading sulfur electrodes, then promoting the formation of nucleation centers at the transition point in discharge will be an effective way of mediating the high-energy barrier. Potential strategies to lower the barrier include cycling the cells at a higher temperature or using additives with surfaces that can serve as good heterogeneous nucleation centers for $\mathrm{Li}_{2} \mathrm{~S}_{2} / \mathrm{Li}_{2} \mathrm{~S}$. Theoretical modeling suggested that $\mathrm{TiS}_{2}$ might have strong interaction with polysulfide species and it has been shown to enhance the power capability of sulfur electrodes [29]. The reason that $\mathrm{TiS}_{2}$ works well as an additive for sulfur electrodes might also be partially related to the enhancement of the $\mathrm{Li}_{2} \mathrm{~S}_{2} / \mathrm{Li}_{2} \mathrm{~S}$ nucleation process. In the efforts to enhance the performance of high loading sulfur electrodes, it is found that $\mathrm{TiS}_{2}$ is indeed an effective additive for this purpose. As it is shown in Figure $6 \mathrm{~g}$, the sulfur electrode at loading level of $4.23 \mathrm{mg} \mathrm{cm}^{-2}$ with $\mathrm{TiS}_{2}$ additive can be discharged at $0.62 \mathrm{~mA} \mathrm{~cm}^{-2}(\sim \mathrm{C} / 11$ rate) without losing the plateau discharge region and still deliver a high level (40\%) of sulfur utilization and an areal capacity of $2.89 \mathrm{mAh} \mathrm{cm}^{-2}$, which is hardly achieved in sulfur electrodes without additives at similar loading level $4.27 \mathrm{mg} \mathrm{cm}^{-2}$ (Figure $6 \mathrm{~h}$ ). The improvement in sulfur utilization is also evident in cell cycling, as shown in Figure 6i, which describes the comparison of the cycling performance. Since in this case a similar sulfur loading and the same electrolyte amount were used, the extent of polysulfide concentration increase during sulfur lithiation will also be similar. It is unlikely that Li-ion transport in electrolyte was the limiting factor for high loading sulfur electrodes. Therefore, methods to accelerate the $\mathrm{Li}_{2} \mathrm{~S}_{2} / \mathrm{Li}_{2} \mathrm{~S}$ nucleation process are promising pathways for the successful development Li-S battery technology with high sulfur loading electrodes.

\section{Conclusion}

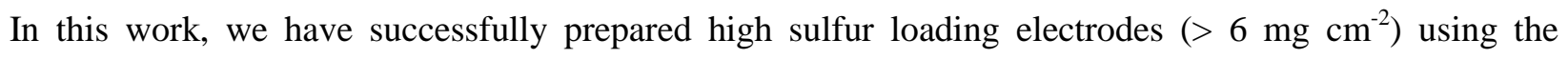
conventional slurry casting process by incorporating a new binder and a new type of microporous carbon. The areal delivered capacity of up to $12 \mathrm{mAh} \mathrm{cm}^{-2}$ has been achieved under low rate coin cell discharge.

E-mail: hgan@bnl.gov 
The carbon particle size and its specific pore volume are identified as critical factors for high sulfur loading electrode preparation and for achieving high sulfur utilization during the Li-S battery electrochemical testing.

The binder hydroxypropyl cellulose (HPC) from the cellulose family has been identified to offer better adhesion between the electrode and the aluminum current collector compared to the commonly used binder polyvinylidene fluoride, PVDF. Because HPC is compatible with nonaqueous solvent NMP, it can reduce the hazards of inclusion of residue water content when the aqueous process is used. Although HPC does slightly increase the resistivity of the electrode network, it serves as an auxiliary binder to help improve the mechanical stability of thick and high sulfur loading electrodes.

In the meantime, 3 different micron-sized microporous carbons derived from natural resources have been studied as sulfur carrier for sulfur electrodes. We find that A5597 has the strongest sulfur absorbing ability during thermal fusion and also endows the electrode with the highest sulfur utilization in Li-S cell test. High specific pore volume instead of specific surface area has been identified as the main parameter that determines A5597's superior property over the other two. This high pore volume is probably related to the special texture of wood (A5597) compared to coconut shell (A5562) and coal (A5583). Wood seems to be the best precursor of microporous carbon for Li-S battery application amongst the three.

Although small particle size sulfur absorbing carbon such as Ketjenblack is still the best choice when power and sulfur utilization are considered as the most important parameters, it is very difficult to prepare thick sulfur electrodes that are mechanically stable with these small particle size carbon candidates such that high energy density can be achieved at the cell level. Bigger microporous carbon particles such as A5597 provide an alternative choice for sulfur incorporation in thick electrodes because of the facility in preparing thick sulfur electrodes with micron-sized particles.

At the moment, high sulfur loading electrodes prepared with the A5597 carbon and binary binder system can only perform as required at low rate, and the kinetic barriers lie in both the transport of Liion/electron and the nucleation process of $\mathrm{Li}_{2} \mathrm{~S}_{2} / \mathrm{Li}_{2} \mathrm{~S}$ at the onset of the discharge plateau region. Improvement on both aspects is necessary before this concept is practical for real applications. Research on microporous carbons with similar or even higher pore volume and primary particle size above 1 micron should also be encouraged for high loading sulfur electrode study. $\mathrm{TiS}_{2}$ additive has also been demonstrated in this work to facilitate the $\mathrm{Li}_{2} \mathrm{~S}_{2} / \mathrm{Li}_{2} \mathrm{~S}$ nucleation and the overall performance of sulfur electrode. Future work is underway to optimize this system to achieve the ultimate goal of efficient highloading sulfur electrodes.

Acknowledgement: This work is supported by the U.S. Department of Energy (DOE) Office of Energy Efficiency and Renewable Energy under the Advanced Battery Materials Research (BMR) program, Contract No. DE-SC0012704. Part of this work has been carried out at the Center for Functional Nanomaterials, Brookhaven National Laboratory, which is supported by the DOE, Office of Basic Energy Sciences, under contract DE-SC0012704. Jian Huang was supported by the U.S. Department of Energy, Office of Science, Office of Workforce Development for Teachers and Scientists (WDTS) under the Science Undergraduate Laboratory Internships Program (SULI).

E-mail: hgan@bnl.gov 


\section{References}

[1] P.G. Bruce, S.A. Freunberger, L.J. Hardwick, J.M. Tarascon, Nat. Mater. 11 (2012) 19-29.

[2] A. Manthiram, Y.Z. Fu, S. H. Chung, C.X. Zu, Y.S. Su, Chem. Rev. 114 (2014) 11751-11787.

[3] S.S. Zhang, J. Power Sources 231 (2013) 153-162.

[4] M. Cuisinier, C. Hart, M. Balasubramanian, A. Garsuch, L.F. Nazar, Adv. Energy Mater. 5 (2015) 1401801-1401806.

[5] X.L. Ji, K.T. Lee, L.F. Nazar, Nat. Mater. 8, (2009) 500-506.

[6]R. Elazari, G. Salitra, A. Garsuch, A. Panchenko, D. Aurbach, Adv. Energy Mater. 23 (2011) 56415644.

[7] H.L. Wang, Y. Yang, Y.Y. Liang, J.T. Robinson, Y.G. Li, A. Jackson, Y. Cui, H.J. Dai, Nano lett. 11 (2011) 2644-2647.

[8] J.C. Guo, Y.H. Xu, C.S. Wang, Nano lett. 11 (2011) 4288-4294.

[9] W.Y. Li, Z. Liang, Z.D. Lu, H.B. Yao, Z.W. Seh, K.Yan, G.Y. Zheng, Y. Cui, Adv. Energy Mater. 5 (2015) 1502539-1502546.

[10] G.M. Zhou, E. Paek, G.S. Hwang, A. Manthiram, Adv. Energy Mater. 6 (2016) 1501355-1501363.

[11] R.D. Cakan, M. Morcrette, F. Nouar, C. Davoisne, T. Devic, D. Gonbeau, R. Dominko, C. Serre, J.M. Tarascon, J. Am. Chem. Soc. 133 (2011) 16154-16160.

[12] J.M. Zheng, J. Tian, D.X. Wu, M. Gu, W. Xu, C.M. Wang, F. Gao, M.H. Engelhard, J.

G. Zhang, J. Liu, J. Xiao, Nano Lett. 14 (2014) 2345-2352.

[13] V. Lapornik, N.N. Tusar, A. Ristic, R.K. Chellappan, D. Foix, R. Dedryvere, M. Gaberscek, R. Dominko, J. Power Sources 274 (2015) 1239-1248.

[14] D. Eroglu, K.R. Zavadil, K.G. Gallagher, J. Electrochem. Soc. 162 (2015) A982-A990.

[15] Z. Yuan, H.J. Peng, J.Q. Huang, X.Y. Liu, D.W. Wang, X.B. Cheng, Q. Zhang, Adv. Funct. Mater. 24 (2014) 6105-6112.

[16] L.X. Miao, W.K. Wang, K.G. Yuan, Y.S, Yang, A.B. Wang, Chem. Commun. 50 (2014) 1323113234.

[17] S.T. Lu, Y. Chen, X.H. Wu, Z.D. Wang, Y. Li, Sci. Rep. 4 (2013) 4629-4629.

[18] G.M. Zhou, L. Li, C.Q. Ma, S.G. Wang, Y. Shi, N. Koratkar, W.C. Ren, F. Li, H.M. Cheng, Nano Energy 11 (2015) 356-365.

[19] G. Hu, C. Xu, Z. Sun, S. Wang, H.M. Cheng, F. Li, W. Ren, Adv. Mater. 28 (2016) 1603-1609.

[20] L. Qie, A. Manthiram. ACS Energy Lett. 1 (2016) 46-51.

[21] K. Sun, H. Liu, H. Gan, J. Electrochem. Energy Convers. Stor., 13 (2016) 021002.

E-mail: hgan@bnl.gov 
[22] J. Christensen, D. Cook, P. Albertus, J. Electrochem. Soc. 160 (2013) A2258-A2267.

[23] M. Smith, R. E. García, Q. C. Horn, J. Electrochem. Soc. 156 (2009) A896-A904.

[24] D.P. Lv, J.M. Zheng, Q.Y. Li, X. Xie, S. Ferrara, Z.M. Nie, L.B. Mehdi, N.D. Browning, J.G. Zhang, G.L. Graff, J. Liu, J. Xiao, Adv. Energy Mater. 5 (2015) 1402290-1402297.

[25] Y.W. Ma, H.Z. Zhang, B.S. Wu, M.R. Wang, X.F. Li, H.M. Zhang, Sci. Rep. 5 (2015) 14949.

[26] M. He, L.X. Yuan, W.X. Zhang, X.L. Hu, Y.H. Huang, J. Phys. Chem. C 115 (2011) 15703-15709.

[27] R.J. Pugh, T. Matsunaga, F.M. Fowkes, Colloids Surf., 7 (1983) 183-207.

[28] K. Yang; Q. M. Gao; Y. L. Tan; W. Q. Tian; L. H. Zhu; C. X. Yang, Microporous and Mesoporous Materials 204 (2015) 235-241.

[29] Z.W. Seh, J.H. Yu, W. Li, P.C. Hsu, H. Wang, Y. Sun, H. Yao, Q. Zhang, Y. Cui, Nat.

Commun. 5 (2014) 5017.

zE-mail: hgan@bnl.gov 
Table 1. Electrode Composition Table

\begin{tabular}{|c|c|c|c|c|c|c|c|}
\hline \multirow{2}{*}{ Composition } & \multirow{2}{*}{ Study } & \multicolumn{6}{|c|}{ Weight Percentage $(\%)$} \\
\hline & & Sulfur & $\begin{array}{c}\text { Microporous } \\
\text { carbon }\end{array}$ & Super C65 & $\mathrm{TiS}_{2}$ additive & HPC binder & PVDF binder \\
\hline 1 & Binder selection & 50 & & 40 & & 10 & \\
\hline 2 & Binder selection & 50 & & 40 & & & 10 \\
\hline 3 & Carbon selection & 62 & $21^{*}$ & 9 & & 8 & \\
\hline 4 & Loading effect & 62 & $27 * *$ & 3 & & 4 & 4 \\
\hline 5 & Loading mechanism & 56 & $16^{* *}$ & 11 & 9 & 4 & 4 \\
\hline
\end{tabular}

* All types of carbon; ** A5597 only.

Table 2. Current Density and C-Rate for Different Sulfur Loading Electrode

\begin{tabular}{|c|c|c|c|c|c|}
\hline \multirow{2}{*}{$\begin{array}{l}\text { Sulfur Loading } \\
\text { (mg cm-2) }\end{array}$} & \multicolumn{5}{|c|}{ Current Density and C-Rate from Cycle 1 to Cycle 5} \\
\hline & $0.08 \mathrm{~mA} \mathrm{~cm}^{-2}$ & $0.08 \mathrm{~mA} \mathrm{~cm} \mathrm{~cm}^{-2}$ & $0.16 \mathrm{~mA} \mathrm{~cm}-2$ & $0.31 \mathrm{~mA} \mathrm{~cm}^{-2}$ & $0.63 \mathrm{~mA} \mathrm{~cm} \mathrm{~cm}^{-2}$ \\
\hline 2.43 & $\mathrm{C} / 52$ & $\mathrm{C} / 52$ & $\mathrm{C} / 26$ & $\mathrm{C} / 13$ & $\mathrm{C} / 6.5$ \\
\hline 2.51 & $\mathrm{C} / 53$ & $\mathrm{C} / 53$ & $\mathrm{C} / 27$ & $\mathrm{C} / 13$ & $\mathrm{C} / 6.7$ \\
\hline 4.27 & $\mathrm{C} / 91$ & $\mathrm{C} / 91$ & $\mathrm{C} / 45$ & $\mathrm{C} / 23$ & $\mathrm{C} / 11$ \\
\hline 4.48 & $\mathrm{C} / 95$ & $\mathrm{C} / 95$ & $\mathrm{C} / 48$ & $\mathrm{C} / 24$ & $\mathrm{C} / 12$ \\
\hline 6.48 & $\mathrm{C} / 138$ & $\mathrm{C} / 138$ & $\mathrm{C} / 69$ & $\mathrm{C} / 34$ & $\mathrm{C} / 17$ \\
\hline 7.24 & $\mathrm{C} / 154$ & $\mathrm{C} / 154$ & $\mathrm{C} / 77$ & $\mathrm{C} / 39$ & $\mathrm{C} / 19$ \\
\hline 8.9 & $\mathrm{C} / 189$ & $\mathrm{C} / 189$ & $\mathrm{C} / 95$ & $\mathrm{C} / 47$ & $\mathrm{C} / 24$ \\
\hline 10.32 & $\mathrm{C} / 219$ & $\mathrm{C} / 219$ & $\mathrm{C} / 110$ & $\mathrm{C} / 55$ & $\mathrm{C} / 27$ \\
\hline
\end{tabular}



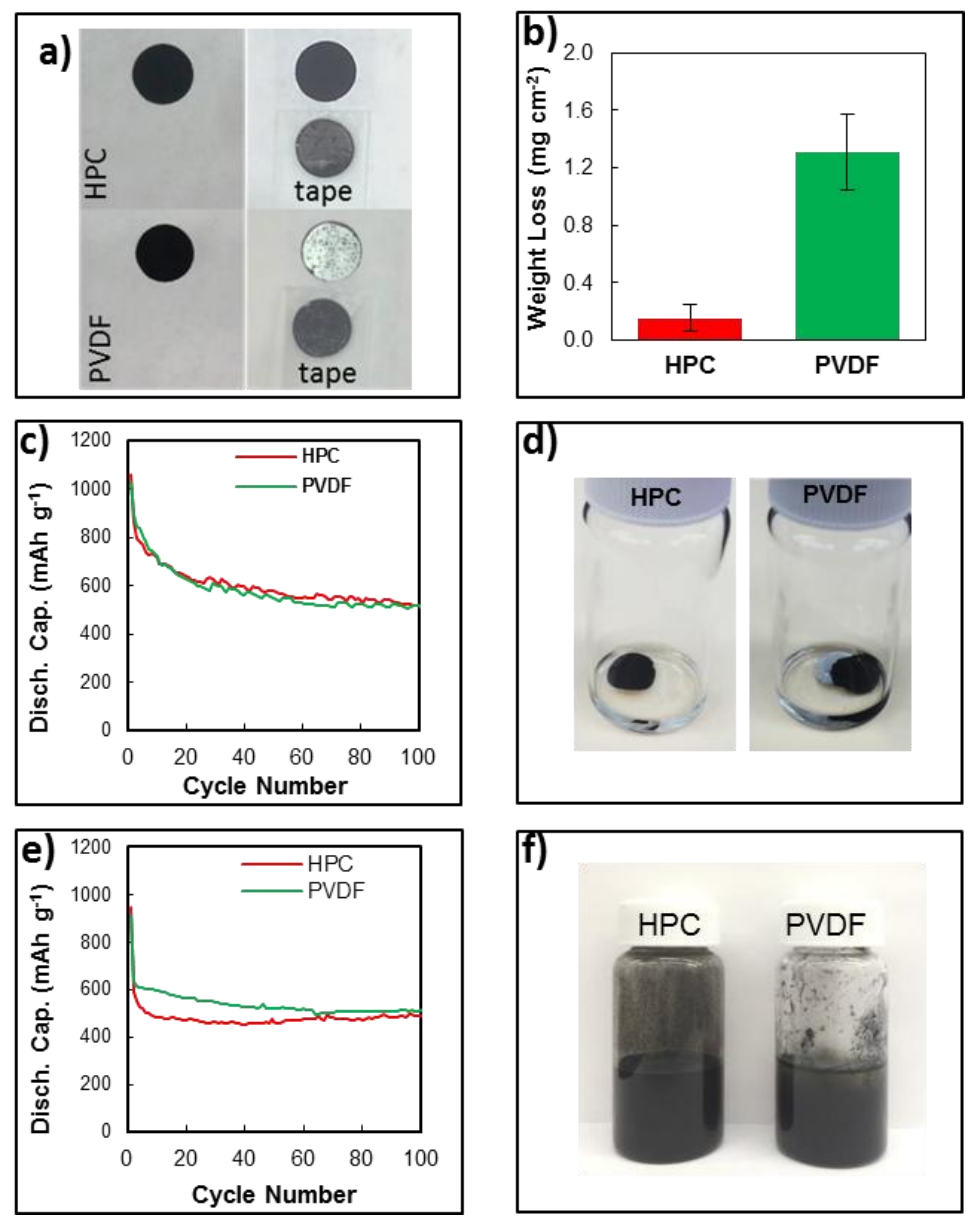

Figure 1. a) Photographs of sulfur electrodes with HPC and PVDF binders before and after tape test; b) average weight loss per area of sulfur electrodes, average loading before tape test: $1.8 \mathrm{mg} \mathrm{cm}-2$; c) cycling performance of sulfur electrodes at $0.2 \mathrm{C}$ between 1.8 and $2.6 \mathrm{~V}$; d) photographs of sulfur electrodes after 100 cycles shown in c; e) cycling performance of sulfur electrodes at $1 \mathrm{C}$ between $1.8 \mathrm{~V}$ and $2.6 \mathrm{~V}$; f) sulfur electrode slurries with HPC and PVDF binder, after $1 \mathrm{hr}$ of settling.
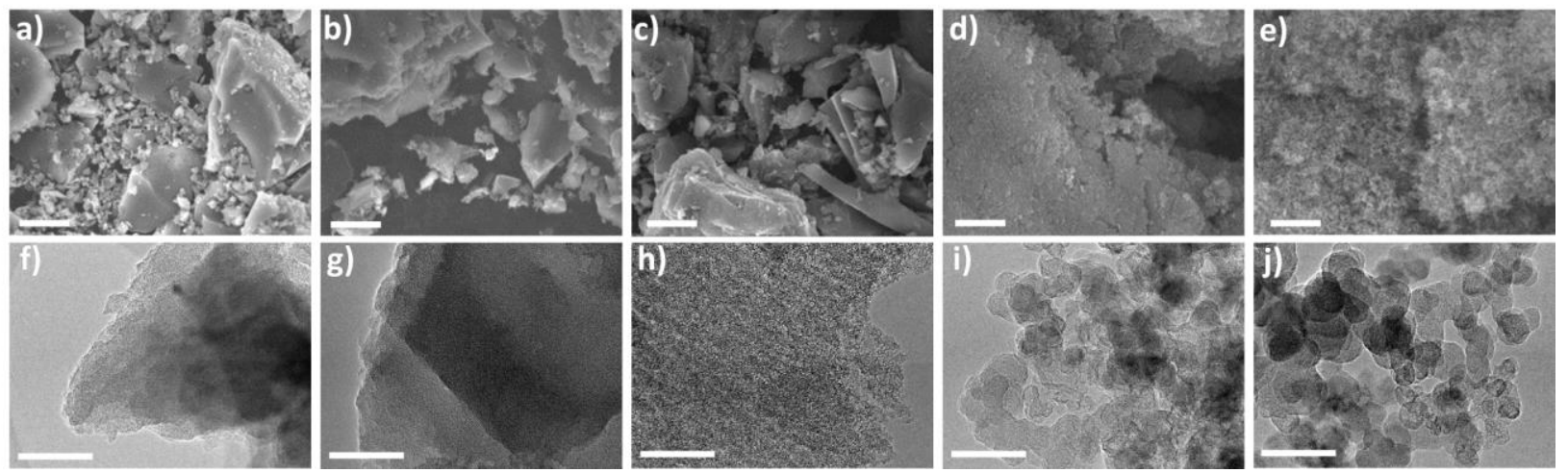

Figure 2. a)-e) SEM micrographs of A5562, A5583, A5597, Ketjen Black and Super C65, respectively, scale bar $=10 \mu \mathrm{m}$; f)-j) TEM micrographs of A5562, A5583, A5597, Ketjen Black and Super C65, respectively, scale bar $=100 \mathrm{~nm}$. 

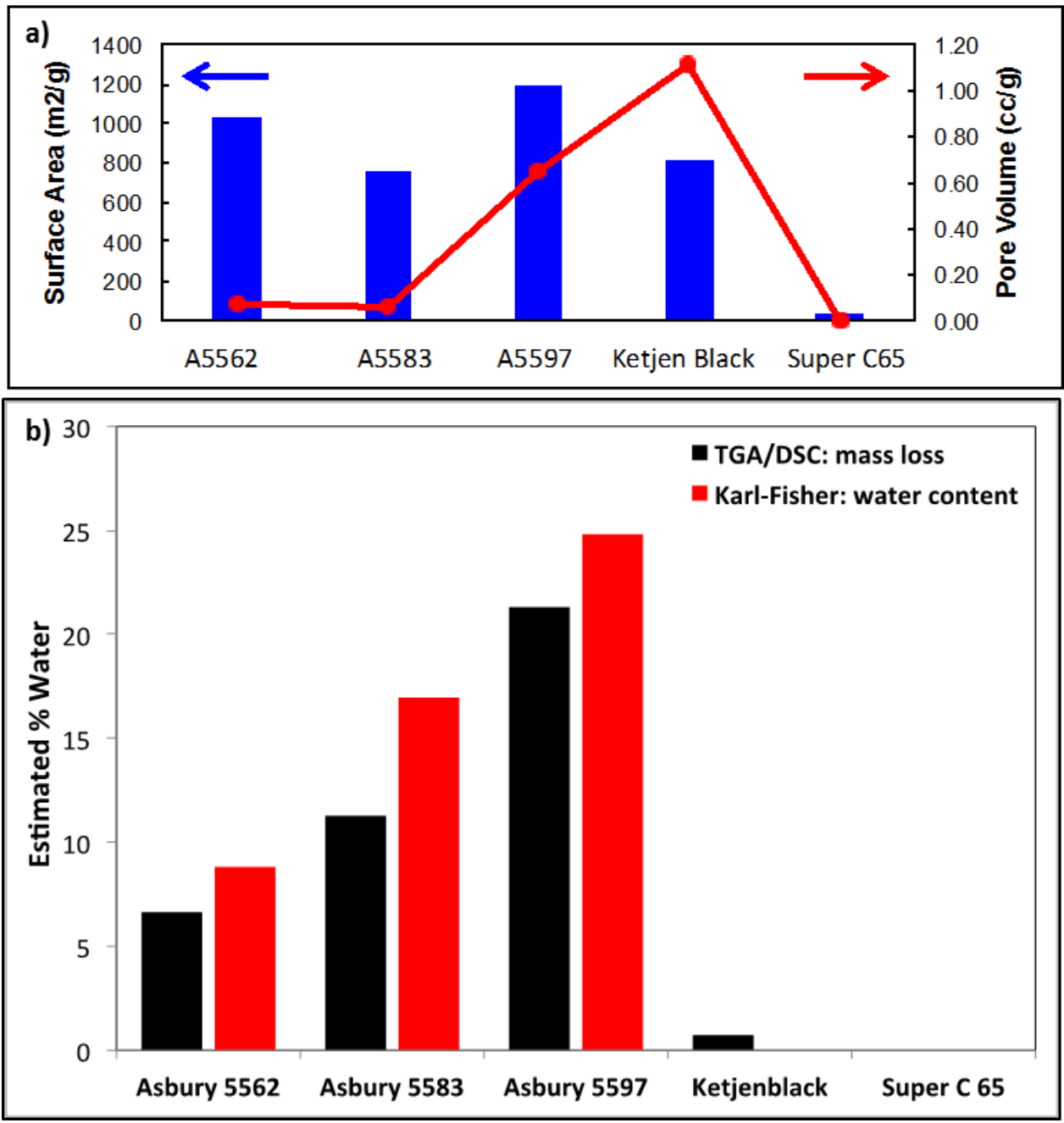

Figure 3. a) BET surface area and specific pore volume of different carbon; b) water absorbed by carbon - TGA weight loss and water content by Karl-Fisher. 

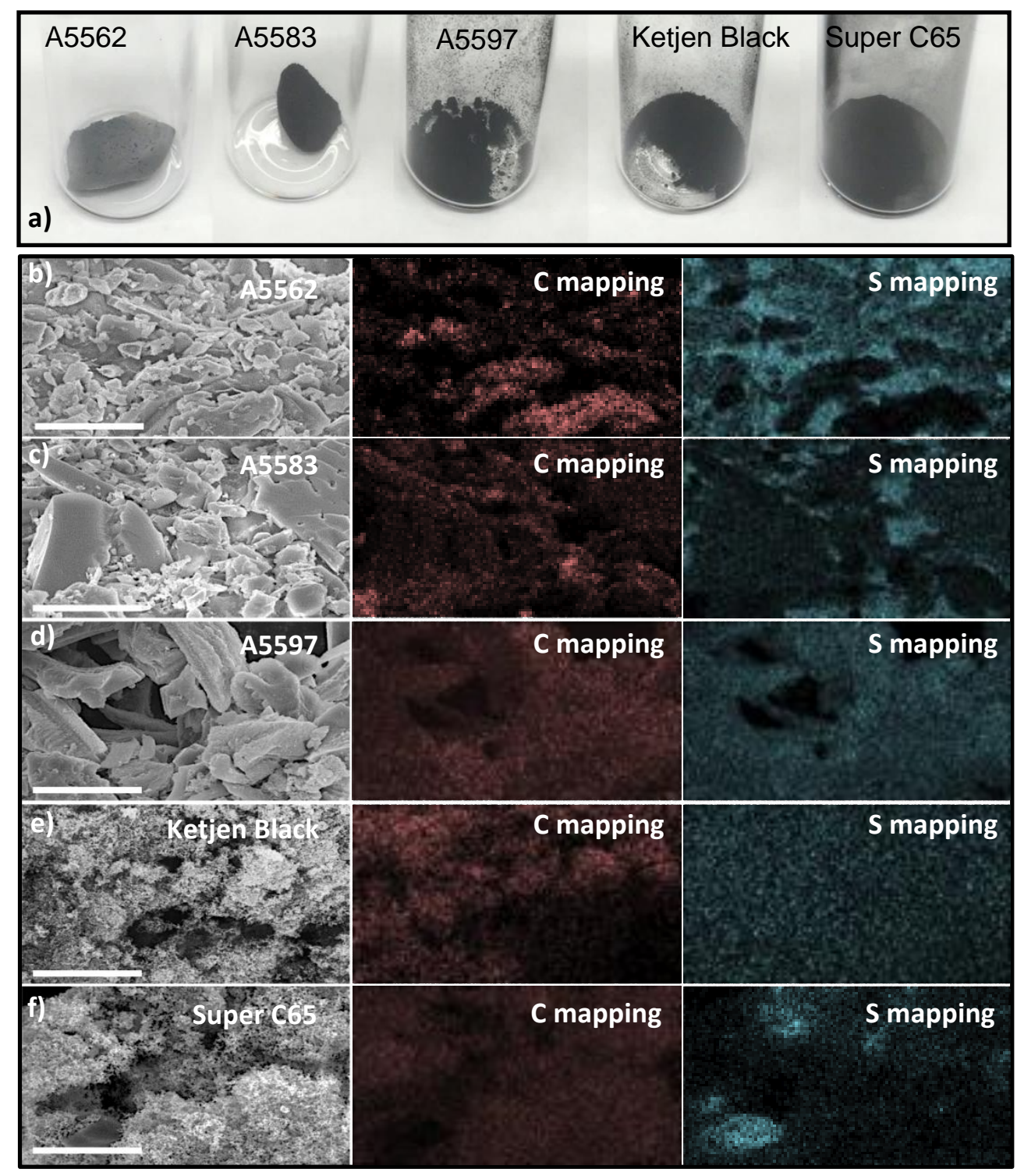

Figure 4. a) Morphology of different S-Carbon mixture after thermal fusion process; c)-f) SEM micrographs of S-Carbon mixture after thermal fusion derived from A5562, A5583, A5597, Ketjen Black and Super C65 respectively, corresponding carbon and sulfur EDS mapping are shown next to them from left to right Scale Bar $=10$ um. 

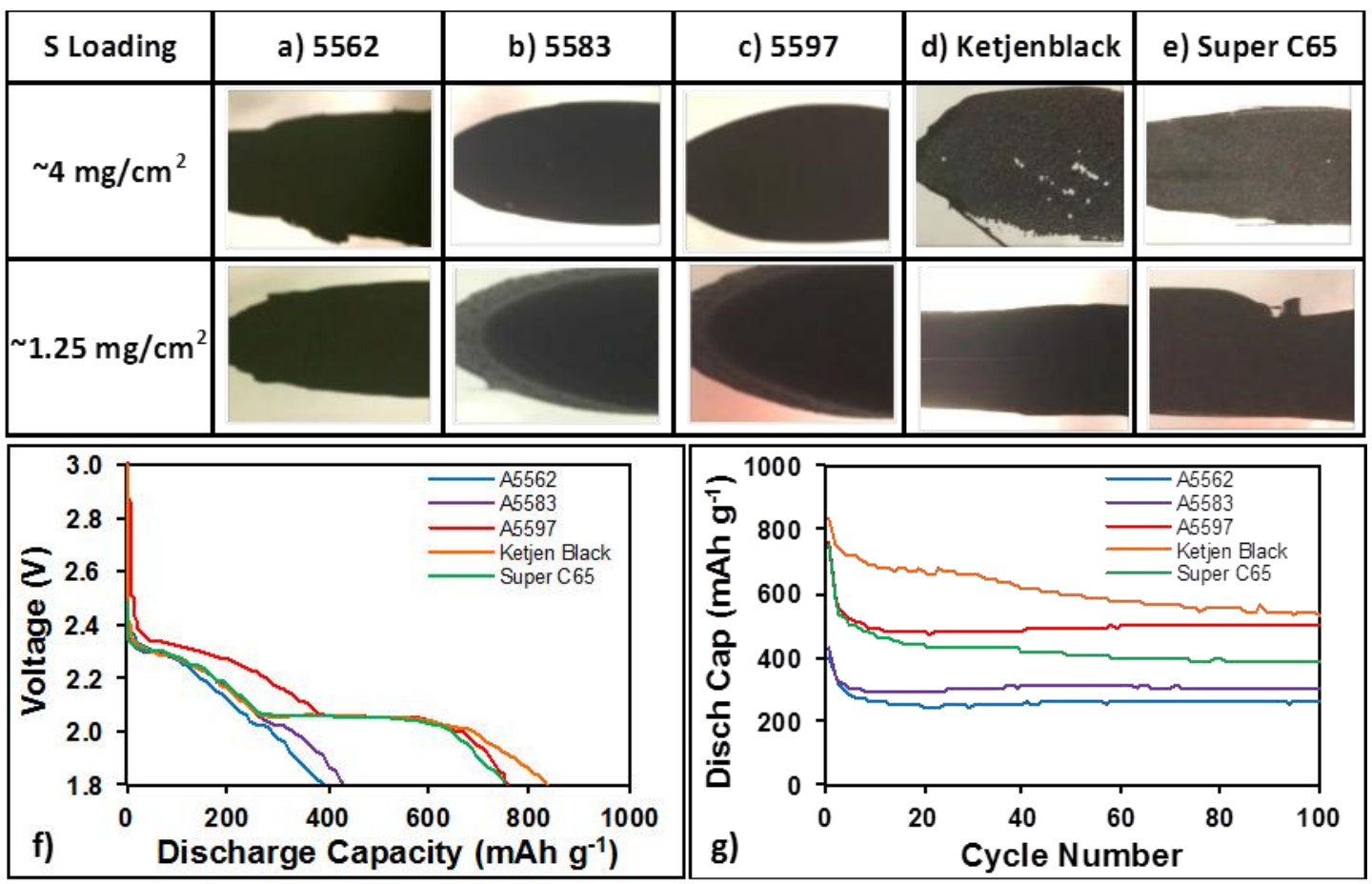

Figure 5. a) - e) Photographs of sulfur electrodes with A5562, A5583, A5597, Ketjen Black and Super C65 as carbon matrix respectively, upper photos electrode loading $\sim 4 \mathrm{mg} \mathrm{cm}^{-2}$ of sulfur, lower photos electrode loading $\sim 1.25 \mathrm{mg} \mathrm{cm}^{-2}$ of sulfur ; f) first discharge voltage profile of the 5 different electrodes at $\mathrm{C} / 5$, loading $\sim 1.25 \mathrm{mg} \mathrm{cm}^{-2}$ of sulfur; g) cycling performance of 5 different electrodes at $\mathrm{C} / 5$, loading $\sim 1.25 \mathrm{mg} \mathrm{cm}^{-2}$ of sulfur. 

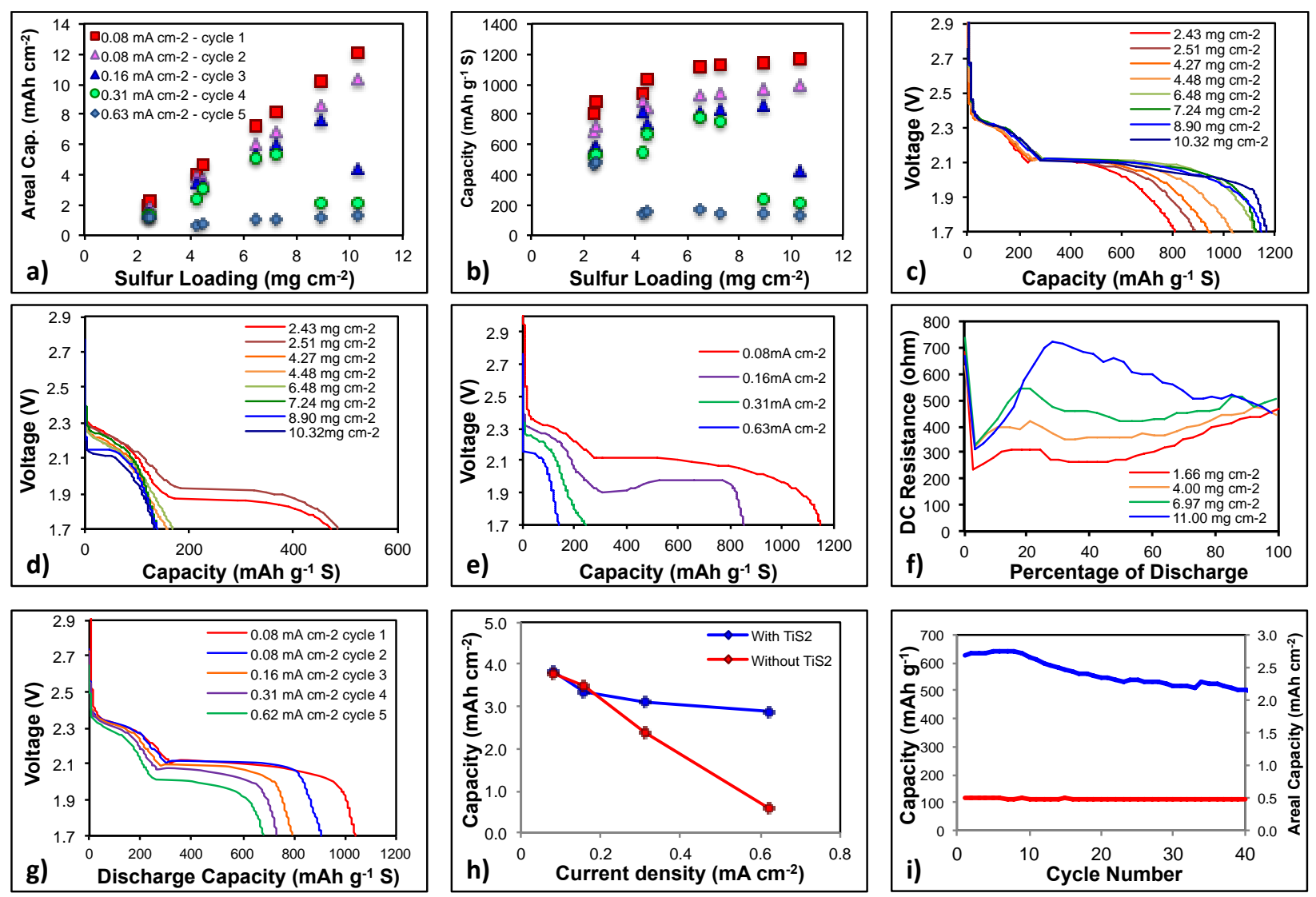

Figure 6. a) Areal discharge capacity and b) sulfur based specific capacity as a function of both the sulfur loading and current density; voltage profiles of electrodes at different levels of sulfur loading at current density of c) $0.08 \mathrm{~mA} \mathrm{~cm}$ and d) $0.63 \mathrm{~mA} \mathrm{~cm}$; e) voltage profiles of sulfur electrode with sulfur loading of $8.90 \mathrm{mg} \mathrm{cm}^{-2}$ at different current densities; f) DC resistance as a function of percentage of discharge for Sulfur electrodes with 4 different loading levels; g) voltage profiles of S-TiS ${ }_{2}$ electrode discharged at different current densities; (h) capacity delivered by S-TiS 2 hybrid electrode $\left(4.23 \mathrm{mg} \mathrm{cm}^{-2}\right.$ sulfur loading) and $\mathrm{S}$ electrode with similar loading $\left(4.27 \mathrm{mg} \mathrm{cm}^{-2}\right.$ sulfur loading) at different levels of current densities; (i) cycling comparison of S-TiS ${ }_{2}$ hybrid and $\mathrm{S}$ electrodes at $0.63 \mathrm{~mA} \mathrm{~cm}^{-2}$. 